\title{
L'immigration d'expression française et la mutation identitaire des organismes de la communauté acadienne et francophone de l'Île-du-Prince-Édouard ${ }^{1}$
}

\author{
Carlo Lavoie \\ Université de l'Île-du-Prince-Édouard
}

L'Acadie est une communauté mémorielle qui n'a jamais atteint un niveau suffisant d'institutionnalisation pour pouvoir effacer sa mémoire, pour pouvoir se penser dans une pure immanence. Sans État, sans territoire - et depuis quelque temps sans Église - l'Acadie se nourrit de sa mémoire, elle en fait le lien par excellence de son unicité (Thériault 2007, 117-118).

L'immigration de langue française est un phénomène relativement nouveau dans les communautés de langue française en situation minoritaire au Canada. Non pas qu'il n'y ait jamais eu d'immigration de langue française dans ces communautés, mais le ralentissement, voire même le déclin de la croissance démographique des francophones fait en sorte qu'il faille maintenant s'y intéresser d'une manière urgente. Par exemple, la communauté acadienne et francophone de l'île-du-Prince-Édouard accuse un retard sur la croissance démographique de l'ensemble de la province, mais elle accuse encore un plus grand retard face à l'ensemble du Canada. Pour faire face à cette problématique, le Carrefour d'Intégration Rurale Évangéline fut créée en 2006 avec un mandat régional avant de devenir, en 2010, un organisme provincial, soit la Coopérative d'intégration francophone (CIF). Depuis 2012, la CIF coordonne le Réseau en intégration francophone de l'Île-du-Prince-Édouard (RIF), un regroupement d'organismes et d'institutions qui s'engagent à appuyer de façon concrète la communauté acadienne et francophone de l'Île-du-Prince-Édouard à accroître sa population. Cette approche permet à la communauté acadienne et francophone de l'Île-du-Prince-Édouard (CAFÎPÉ) de mettre en œuvre une nouvelle stratégie de croissance démographique qui englobe l'immigration, la

\footnotetext{
${ }^{1}$ L'auteur désire remercier Voix vers la Prospérité et UPEI pour leur soutien financier envers ses recherches. Cet article est en partie tiré de recherches subventionnées par Voix vers la prospérité en vertu d'une subvention de partenariat du CRSH (Lavoie et. al. 2016). La Coopérative d'intégration de l'Île-du-Prince-Édouard a également joué un rôle important dans ces recherches en permettant à l'auteur d'élaborer un rapport sur les activités du Réseau d'intégration de l'Île (Lavoie 2015), rapport dont les principales conclusions et recommandations seront en partie discutées dans cet article.
} 
Alternative Francophone vol. 2, 2(2018) : 29-47

http://ejournals.library.ualberta.ca/index.php/af

migration intra-canadienne, le rapatriement, la refrancisation et la francophilie. Beaucoup d'efforts sont déployés, tant par les employés que par des bénévoles des organismes membres du RIF afin de concrétiser cette stratégie de renouvellement démolinguistique. Comme le note le rapport final Transmission de la langue française aux jeunes francophones en situation minoritaire. Théorie et stratégie de Patrimoine canadien, «le renouvellement démolinguistique des CLOSM apparaît comme un facteur essentiel. Il se retrouve en quelque sorte à la base de la vitalité des communautés : sans effectif démographique, la minorité n'existe plus » (Johnson 2015, 2). Cependant, miser en partie sur l'immigration d'expression française pour concrétiser ce renouvellement démolinguistique exige non seulement une nouvelle stratégie, mais un réalignement des discours englobant cette stratégie afin de tenir compte d'une diversité culturelle, économique, sociale et linguistique qui se révèle.

Dans un rapport de recherche publié en 2016 (Lavoie et. al.), nous faisions mention de la difficulté, non seulement d'obtenir des données objectives en matière d'immigration francophone, mais de les utiliser sans crainte d'identifier directement les personnes concernées. Car, il faut avouer qu'il n'y a pas beaucoup d'immigrants d'expression française sur l'île et ceux que nous y retrouvons sont, pour le moins que l'on puisse dire, suffisamment connus pour être reconnus par de brèves descriptions. Ainsi, le rapport de la direction de la Coopérative d'intégration francophone (CIF) faisait état de 32 clients adultes et 13 enfants qui avaient profité des offres au niveau de l'intégration en 2014-2015 (2015, 15). Cependant, puisqu'ils s'insèrent dans une petite communauté d'environ 5000 francophones, leur nombre, même petit, revêt une importance capitale (Cormier 2013). Ainsi, sans même le demander ni même le vouloir, le nouvel arrivant se voit la source d'une mutation identitaire des organismes de la communauté acadienne et francophone de l'Île-du-Prince-Édouard. Ce sont ces répercussions involontaires, sorte d'effets collatéraux sur le sentiment d'appartenance et l'habitus collectif, que nous proposons d'explorer ici. Notre étude portera sur le devenir-hybride des symboles utilisés pour décrire la CAFÎPÉ en lien avec un régime identitaire qui se veut moderne et le pacte proposé dans le recrutement et l'intégration de nouveaux arrivants d'expression française sur l'Île. Nous nous intéresserons particulièrement aux éléments historiques et discursifs mettant au jour une transformation de l'identité des organismes de la communauté acadienne et francophone avec, en toile de fond, un imaginaire collectif oscillant entre le désir naturel de repli sur soi-même et le désir d'inclusion de l'Autre motivé par la nécessité.

\section{Appartenance et habitus : un désir de faire-société}

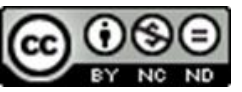


Alternative Francophone vol. 2, 2(2018) : 29-47

http://ejournals.library.ualberta.ca/index.php/af

Ainsi, la question de l'intégration du nouvel arrivant est intimement liée à la notion d'appartenance qui permet de désigner un groupe primaire à l'intérieur duquel chaque membre se (re) connaît et bénéficie de relations directes. C'est par l'appartenance à un groupe que nous arrivons à participer à une vie collective qui nous transmet nos valeurs, nos règles de conduite et même nos stéréotypes. L'appartenance nous demande de nous plier devant une pression de conformité à laquelle nous obéissons en général. Je vis comme les autres membres de mon groupe : "Un pays, un drapeau, un hymne [...] tous pour un », comme dirait la narratrice du roman La Québécoite de Régine Robin $(1983,6)$. Toutefois, un conflit surgit chez l'individu en face de valeurs, normes, critères de jugement et de conduite, idées et idéaux d'un autre groupe (appelé alors groupe de référence) représentant d'autres aspirations personnelles ou morales. L'individu se retrouve alors aux prises avec une différence par rapport à l'Autre, une position d'« être-différent » avec laquelle il faudra inévitablement « faire sens » à l'intérieur d'une structure sociale donnée d'où naittra une idée structurante, soit le sentiment d'appartenance. Entre ainsi en jeu ce que Pierre Bourdieu nomme l'« habitus », soit un "principe [...] unificateur qui retraduit les caractéristiques $[\ldots]$ relationnelles d'une position en $[\ldots]$ un ensemble $[\ldots]$ de choix [...] qui met en œuvre des principes de différenciation» (Bourdieu 1979, 24). Cet ensemble de choix, issu d'une diversité et d'une multiplicité

de l'ensemble des pratiques accomplies dans des champs dotés de logiques différentes, [...] fait disparaitre aussi la structure de l'analyse symbolique qui dessine l'ensemble de ces pratiques structurées, dans tous ces styles de vie distinctifs qui se définissent toujours objectivement et parfois subjectivement dans et par leurs relations. (Bourdieu, 1979: 112)

Dans le cas des communautés de langue officielle en situation minoritaire, cette multitude de champs de logiques différentes présente une tension entre le groupe «dominant » et un groupe plus petit, minoritaire, vu comme « dominé » (François 2007, 45). Pour l'individu, se pose alors une question existentielle: comment habiter un territoire sous cette diversité et multiplicité de pratiques dotées de logiques différentes? Au niveau du groupe minoritaire se poserait alors la question du «faire-société ». Cette question, comme le montre Joseph Yvon Thériault, problématise «les questions de la mémoire, de la tradition, de l'histoire » (6). Elle apparaît essentielle dans « le maintien de la tension entre [la diversité de l'] identité et [la multiplicité des pratiques inhérentes à la] modernité » (6). Les lieux de mémoire, facteurs importants dans la constitution d'une communauté, proposent au groupe civil qui nous intéresse particulièrement ici, le projet de « faire œuvre de civilisation en français dans le continent anglo-américain» (8). Ce projet pourra difficilement devenir 
Alternative Francophone vol. 2, 2(2018) : 29-47

http://ejournals.library.ualberta.ca/index.php/af

opératoire dans l'ombre de la communauté économique dominante, la communauté anglophone, sans créer de tensions au niveau identitaire. Penser ainsi l'Acadie comme une société civile permet, pour reprendre les mots de Thériault qui applique cette notion à l'ensemble de la francophonie canadienne, d'envisager un lieu hors État qui n'est pas pour autant un espace de libre déploiement des individualités et des intérêts privés [...]. La société civile a une dimension politique, bien que non étatique. L'Acadie comme société civile permet de rendre compte de l'historicité de groupement d'individus qui transcende les générations (11).

C'est en s'inscrivant dans l'histoire que l'Acadien se reconnaît à la fois comme membre d'une ethnie et membre d'une communauté linguistique minoritaire. Cette double appartenance peut devenir source de conflit interne, l'Acadien n'étant plus nécessairement francophone de nos jours. Comment alors inviter le nouvel arrivant de langue française à embrasser cette appartenance et éviter qu'il s'assimile à la majorité anglophone? La notion de mémoire partagée serait alors appelée à jouer un rôle essentiel en lien avec les questions de résistance à l'assimilation et de résilience de la société acadienne, véritables concepts opératoires clés de la conservation d'un patrimoine culturel issu de divers symboles et lieux de mémoire.

\section{Symboles et histoire}

Ainsi, en lien avec l'habitus acadien de l'Île se présente l'histoire acadienne comme continuité des symboles et sémiotique de la mémoire partagée (Paré 2003, 63). Les publications sur les contes, légendes et fêtes populaires de Georges Arsenault (1987, 1998), les recherches portant sur l'histoire (Lockerby 2010) et sur la généalogie de familles acadiennes menées par des historiens tant francophones qu'anglophones, ainsi que les célébrations de moments historiques forts participent de cette sémiotique de la mémoire partagée. Cette sémiotique est aussi présente dans le mouvement associatif de langue française qui regroupe aujourd'hui une cinquantaine d'organismes provinciaux et régionaux qui, ensemble, établissent la gouvernance d'une communauté qui se veut acadienne et francophone ${ }^{2}$. La Société Saint-Thomas d'Aquin (SSTA), fondée en 1919, est le porte-parole officiel, à la fois de ces organismes et «des Acadiens, Acadiennes et francophones de l'̂lle-du-PrinceÉdouard. Son mandat est de travailler pour que tout Acadien, toute Acadienne

\footnotetext{
2 Pour une liste exhaustive des organismes de la communauté acadienne et francophone de l'Île, voir le site Internet de la Société Saint-Thomas-d'Aquin à https://ssta.org/membrespartenaires/partenaires-autres.
} 
Alternative Francophone vol. 2, 2(2018) : 29-47

http://ejournals.library.ualberta.ca/index.php/af

et francophone puisse vivre et s'épanouir individuellement et collectivement en français à l'Île-du-Prince-Édouard » (SSTA, Mission) et de faire société. Son action comporte à la fois un volet de revendication politique auprès des différents gouvernements, la gestion d'un fonds d'aide aux étudiants, la promotion des services et des activités offerts en français et la représentation auprès des autres communautés de langue française.

Le plan de travail en vue du développement global adopté par la SSTA en 2016 met l'accent sur l'importance des six centres scolaires et communautaires de la province, chacun «constituant le seul lieu dynamique dans la plupart des régions susceptibles d'offrir des services et des activités en français » (SSTA 2016, 11). Ces centres sont des écoles dont les locaux servent aussi d'espace communautaire ou qui font partie de complexes qui abritent une école et un centre communautaire dont le but est des rassembler les Acadiens, Acadiennes et francophones de sa région, d'organiser des activités et d'offrir des services en français afin de promouvoir la langue française et la culture acadienne. Le discours officiel émanant de la SSTA affirme que

la présence de ces centres est essentielle afin d'assurer un plus grand épanouissement de la communauté, surtout au niveau régional. Les Acadiens, Acadiennes et francophones sont conscients du rôle primordial de ces centres dans leur ralliement et dans le rayonnement de leur culture, autant auprès des francophones que des anglophones. (SSTA, Comités régionaux de la SSTA)

Par leur ralliement et le rayonnement de la langue et de la culture, ces centres constituent le point central de la résistance à l'assimilation et constituent autant d'espaces habitables à l'intérieur desquels pratiquement toutes les activités en français ont lieu sur l'Île. En plus de l'enseignement offert en français, on y retrouve en certains endroits du théâtre communautaire, des spectacles de groupes et de chanteurs acadiens de l'île, mais aussi du continent et d'artistes québécois, des soirées sociales, des conférences, des formations sur le développement personnel ou encore sur le développement économique régional. En dehors de ces centres, peu d'autres lieux présentent des activités en français sur une base régulière ${ }^{3}$. Étant donné que la mémoire acadienne est la source de l'identité acadienne de l'île, l'on ne sera pas surpris de constater un fort attachement à l'histoire acadienne et à son folklore dans l'espace public communautaire. Mais peut-on parler d'épanouissement communautaire et

${ }^{3}$ Parmi ces lieux, notons le Musée acadien de Miscouche, le site historique Port-Lajoie-FortAmherst, le Village musical acadien d'Abram Village, le Village des sources l'Étoile filante, la Banque des fermiers et la maison Doucet de Rustico. À l'exception du Village des Sources, ces lieux constituent des lieux mémoire privilégiés de l'histoire acadienne de l'Île. 
Alternative Francophone vol. 2, 2(2018) : 29-47

http://ejournals.library.ualberta.ca/index.php/af

personnel en français pour autant? Parler français sur l'Île semble très difficile ailleurs que dans ces lieux repliés sur eux-mêmes et dans l'espace intime de quelques maisons. Le touriste qui se promène dans les rues de Charlottetown sera d'ailleurs surpris par l'absence d'affiches publicitaires en français, d'autant plus que la présence d'affiches publicitaires de langue chinoise est de plus en plus forte.

Malgré tout le chemin parcouru depuis la Renaissance acadienne et les conventions nationales du $19 \mathrm{e}$ siècle et du début du $20^{\mathrm{e}}$ siècle, l'Acadie de l'Île subit les effets d'une difficile rétention de son effectif francophone. Ainsi, le recensement officiel de 1881 comptait 10751 Acadiens sur une population de 108891 habitants, soit environ $10 \%$ de la population insulaire. Au recensement de 2011, la CAFÎPÉ ne comptait plus que 5195 personnes ayant le français comme langue maternelle, soit environ 3,8 \% de la population. Les données du recensement de 2011 nous apprennent également que seulement 2465 francophones parlent français le plus souvent à la maison, pour un taux de

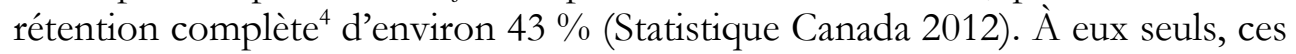
chiffres devraient être suffisants pour convaincre quiconque de l'urgence de la situation. Le salut de la CAFÎPÉ résiderait dans une stratégie de croissance démographique dont les actions visent des résultats tangibles au niveau de l'augmentation :

De la population de langue maternelle française

Du taux de transmission linguistique

Du nombre d'enfants inscrits dans les écoles de langue française

De la francisation de la population acadienne et francophone anglicisée

De la population francophile de l'Île-du-Prince-Édouard

Du nombre d'immigrants francophones à l'île-du-Prince-Édouard

De l'appui des anglophones de l'Île à l'épanouissement de la francophonie Insulaire. (tonile.ca)

Ainsi, la stratégie de croissance démographique de la CAFÎPÉ repose sur l'accroissement du nombre de locuteurs du français à l'Île. Il devient impératif

${ }^{4}$ Nous empruntons ici la terminologie de Statistique Canada pour qui «le terme "rétention" désigne le phénomène selon lequel les personnes ayant une langue maternelle donnée parlent cette langue à la maison. Cette rétention est dite "complète" lorsque cette langue est parlée le plus souvent, et "partielle" lorsqu'elle est parlée régulièrement, bien qu'elle ne soit pas la principale langue d'usage. La notion de "taux de rétention (complète ou partielle)" désigne la proportion de la population d'une langue maternelle donnée qui parle cette langue à la maison, le plus souvent ou régulièrement. Le taux de rétention fournit une indication de la vitalité linguistique des groupes, notamment de l'importance de la transmission des langues entre les générations ». (Statistique Canada 2012)

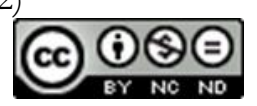


Alternative Francophone vol. 2, 2(2018) : 29-47

http://ejournals.library.ualberta.ca/index.php/af

dans ce contexte de cultiver un sentiment d'appartenance qui repose sur un habitus collectif qui soit partagé non seulement par l'Acadien et l'anglophone insulaire (qu'il soit anglophone, francophile ou acadien anglicisé), mais aussi par le nouvel arrivant de langue française.

\section{Immigration et le Réseau en intégration francophone}

L'approche de concertation mise sur pied par la CAFÎPÉ pour cultiver ce sentiment d'appartement s'effectue par l'entremise du Réseau d'intégration francophone, le RIF, dont la portée s'étend à tous les volets de la croissance démographique, soit l'immigration, la migration, le rapatriement, la refrancisation et la francophilie. Le mandat du RIF d'assurer le développement des activités, des services et des ressources qui favoriseront une augmentation des effectifs démographiques acadiens et francophones de la province en fait un forum de concertation pour ses membres concernant leurs activités en matière de croissance démographique.

Ensemble, les organismes membres du RIF sont issus du mouvement associatif de langue française et œuvrent sur plusieurs fronts qui ont, comme tronc commun, la langue française afin de permettre aux Acadiens et francophones de l'Île de faire société en mettant à l'avant-scène la survivance et la résilience de la communauté. Le discours sur la survivance de la communauté acadienne et francophone propageant l'histoire et le folklore de la culture acadienne se retrouve en concomitance avec un discours sur la résilience qui veut projeter l'Acadie vers le futur à l'aide de ses accomplissements et de ses histoires à succès. Les membres réguliers du RIF représentent les organismes qui ont le plus à gagner de façon directe de la présence de nouveaux arrivants, soit la SSTA, par son plan de développement global, la CIF, dont le mandat est d'accueillir et d'intégrer les nouveaux arrivants de langue française, mais aussi de sensibiliser la population francophone aux bienfaits de l'immigration (rappelons que c'est d'ailleurs la CIF qui est chargée de coordonner le RIF), le Collège de l'île, la Commission scolaire de langue française (CSLF) et la Fédération des parents (FP) dans le but d'élargir leur clientèle étudiante ainsi que le Réseau de développement économique et d'employabilité (RDÉE-ÎPÉ) dont le rôle est de favoriser le développement économique et entrepreneurial en français sur l'île. Ensemble, ces organismes membres réguliers établissent la gouvernance du RIF et jouissent du soutien d'organismes membres observateurs tels l'Université de l'Île-du-Prince-Édouard et différents ministères provinciaux (Bureau de l'immigration, de l'établissement et de la croissance démographique du Ministère de la main d'œuvre et des études supérieures et le Secrétariat aux Affaires 
Alternative Francophone vol. 2, 2(2018): 29-47

http://ejournals.library.ualberta.ca/index.php/af

acadiennes et francophones) et fédéraux (IRCC et Patrimoine Canadien). Nous nous intéressons plus particulièrement ici aux membres réguliers, car ils participent de près à la prise de décision du réseau et leurs discours nous permettent d'identifier deux axes discursifs précis.

Le premier de ces axes mise sur une base linguistique forte et propose une francophonie éclatée à l'intérieur de laquelle il est possible s'intégrer en français. La désignation de « communauté acadienne et francophone » retrouvée dans la mission d'organismes de la CAFÎPÉ tend à céder le pas ici à la désignation de «communauté francophone ». Ainsi, le RDÉE, la CIF, la CSLF, le Collège de l'île et la FP proposent d'abord dans leur nom même un pacte civique en français. Leur mission et mandat s'articulent autour d'une notion linguistique et proposent une Acadie de la francisation basée sur la francophonie et la francophilie, en vantant les vertus du bilinguisme et de la diversité. Si le français prédomine dans leur mandat, c'est pour sauvegarder cette langue, mais aussi pour offrir à l'anglophone la possibilité de devenir bilingue. Le lien avec le francophile se fait par le caractère utilitaire de la langue française devenue un atout, surtout grâce à la présence du Ministère des anciens combattants du Canada à Charlottetown.

Le second axe qui se dégage est celui d'une acadianisation forte, qu'elle soit de langue française ou de langue anglaise. Ainsi, la Société Saint-Thomasd'Aquin, porte-parole de la communauté, se retrouve, malgré sa vision, son mandat et tous ses efforts, à représenter une ancienne Acadie à cause de son nom même qui propose une identification ethnique basée sur la religion ${ }^{5}$. Pourtant, la SSTA reconnait que

les Acadiens et les francophones doivent chercher à intégrer davantage les autres citoyens d'expression française insulaires à l'espace francophone «et que cette intégration » est essentielle à l'épanouissement à moyen et long terme de la francophonie insulaire et selon certains, à sa pérennité. (SSTA 2016, 3)

Outre le nom même de la SSTA, une certaine difficulté de représentation se pose dans la nomenclature même des groupes dont l'organisme se veut le porteparole. L'on veut coordonner les adjectifs «acadiens", "acadiennes » et « francophones » comme si l'on parlait de deux groupes distincts qui ne seraient

\footnotetext{
${ }^{5}$ Il est en effet difficile de faire abstraction de la religion catholique lorsqu'un organisme porte le nom d'un théologien italien du 13 è siècle. Docteur de l'Église, patron des universités, écoles et académies catholiques ainsi que des libraires, Thomas d'Aquin fut canonisé en 1323 et son importance philosophique et théologique pour l'Église catholique fut reconnue suite au concile Vatican II qui fit de son enseignement la base de la formation des prêtres. (Boulogne 1965)
} 
Alternative Francophone vol. 2, 2(2018): 29-47

http://ejournals.library.ualberta.ca/index.php/af

pas perméables. Le désir d'inclusion des francophones crée malgré tout une exclusion en déterminant de façon claire que les francophones ne sont pas pour autant des Acadiens, tout en faisant comprendre que les Acadiens ne seraient pas pour autant des francophones. Avec un taux de rétention du français de $43 \%$, l'on comprend que des Acadiens sont maintenant anglophones. Mais l'on comprend aussi que le francophone n'est pas acadien et qu'il serait condamné à porter le symbole de sa migration en se voyant refuser une identité qu'il pourrait partager avec le natif d'Acadie. En anglais, les Insulaires utilisent la notion de From Away pour désigner toute personne originaire de l'extérieur de l'île. Le francophone serait ainsi originaire de l'extérieur de l'Acadie de l'île. Contrairement à la CIF, au RDEE, à la CSLF et au Collège de l'île, la SSTA tient à cette distinction, tant dans sa vision, sa mission, que dans ses buts. La diversité vue ainsi devient source de catégorisation limitée, binaire, une référence ethnique et minoritaire rappelant, comme le dirait Joseph Yvon Thériault, l'ambivalence d'une communauté en situation minoritaire (172), à l'image de celle portée par la Fédération des communautés francophones et acadienne du Canada. Si le Canada comporte effectivement une communauté acadienne et plusieurs communautés francophones, qu'en est-il de l'île-du-Prince-Édouard? L'adjectif «acadien » ne devrait-il pas en lui-même être suffisant sans que l'on soit obligé d'y coordonner l'adjectif «francophone »? Est-ce la façon trouvée par la communauté pour affirmer ce que Thériault appelle «une modalité particulière d'articulation entre résistance et marginalité » (Thériault 31)?

On remarque, parmi les membres du RIF, l'absence du domaine culturel. Il n'y a ni Fédération culturelle ni regroupement d'auteurs ou d'artistes. En fait, sauf en musique et en arts visuels, il y a peu d'artistes professionnels qui œuvrent en français sur l'île. L'Acadie de l'Île n'a publié que très peu d'écrits de fiction, romans, théâtre ou poésie. Le seul roman publié en français sur l'île a été Placide, l'homme mystérieux à New York de Gilbert Buote en 1904'. Un seul auteur originaire de l'Île, Melvin Gallant, s'est démarqué jusqu'à présent avec plus d'une vingtaine de publications parues au Nouveau-Brunswick dont, entre autres, une série de contes acadiens et deux romans historiques portant sur Michel Haché dit Gallant, l'ancêtre des familles Haché et Gallant des provinces maritimes?. Quelques pièces de théâtre communautaire sont écrites et présentées en français

\footnotetext{
${ }^{6}$ Ce roman a d'abord été publié sous forme de feuilleton par le journal L'Impartial de Tignish entre janvier et août 1904 et réédité en 1999 par les éditions Bouton d'or Acadie.

${ }^{7}$ Notons au passage son roman de 2016, À la Conquête de l'île Saint-Jean, dont l'action se déroule à l'île Saint-Jean (maintenant l'île du Prince-Édouard) de 1720 jusqu'à la déportation des Acadiens de l'île en 1758.
} 
Alternative Francophone vol. 2, 2(2018): 29-47

http://ejournals.library.ualberta.ca/index.php/af

chaque année, mais elles ne sont pas publiées. Même la poésie, à l'exception de quelques tentatives d'Angèle Arsenault et de Jacinthe Laforest, se fait discrète. Pourtant, nous sommes d'avis, comme l'avance François Paré dans Les littératures de l'exiguïté, que les cultures minoritaires recourent plus souvent à l'écriture poétique, que les grandes cultures en nous convoquant, « en tant que minoritaires, à l'expérience éclairante d'un langage revalorisé, réinvesti par l'expérience éclairante de sa marginalité » (2001, 138). Nous ajouterions même que l'écriture de fiction, entre autres la poésie, permet de «dire les lieux et les mémoires, l'intimité et la globalité de cette part d'Amérique qui vit, aime, souffre, écrit et rêve en français » (Beausoleil et Leblanc 1999, 7). Par contre, il existe nombre d'ouvrages liés à l'histoire, à la généalogie, aux traditions populaires et au folklore. Des groupes de musique folk-trad, tels Vishtèn ou encore Ten Strings and a Goat Skin perpétuent de nos jours une tradition musicale en français dont Angèle Arsenault aura été une grande ambassadrice. L'imaginaire collectif semble s'incarner uniquement dans les traditions orales, dont le théâtre et la chanson proviennent, à la constante recherche ou (re) découverte de symboles anciens, au "risque de se figer dans une relation folklorique avec le passé » (Thériault, 117-118). Ainsi, les activités culturelles proposées par la CIF se veulent des activités festives de sensibilisation à la diversité culturelle. L'Insulaire anglophone semble toutefois réticent à participer à ces activités. Bien que 4948 élèves étaient inscrits dans les programmes d'immersion française à travers la province en 2016-2017 (Canadian Parents for French 2016), peu d'activités culturelles sont partagées, rendant encore plus palpable pour les anglophones le fait que le français puisse être une langue de travail et non une langue de culture dans laquelle l'on puisse vivre. Malgré les efforts du RIF, de la CIF, des centres scolaires et communautaires régionaux et de nombreux autres organismes, l'habitus collectif proposé par la CAFÎPÉ semble difficile à percer. Ses activités en français restent en marge de la communauté insulaire en général. Comment dans ce contexte favoriser l'intégration du nouvel arrivant de langue française?

\section{Le point de vue d'immigrants d'expression française}

Pour mieux comprendre les effets liés à l'habitus collectif de l'Acadie de l'île, nous avons cherché à mieux saisir la portée des efforts de la CAFÎPÉ en matière d'intégration des nouveaux arrivants, en particulier les efforts du RIF. Pour ce faire, nous avons utilisé les résultats d'un sondage en ligne que nous avons effectué pour le compte de la CIF auprès des nouveaux arrivants de langue française entre le 15 mars et le 10 avril 2015 (Lavoie 2015). Dans cette optique, la CIF a invité les nouveaux arrivants qui étaient à l'Île depuis une période d'au moins trois mois et un maximum de 60 mois par courriel, Twitter, 
Alternative Francophone vol. 2, 2(2018) : 29-47

http://ejournals.library.ualberta.ca/index.php/af

Facebook et le site Internet tonile.ca. 15 participants âgés d'au moins 19 ans ont répondu à l'appel. Ce sondage était divisé en 6 sections. Outre l'introduction, la section 1 contenait des questions démographiques; la section 2, des questions relatives à l'attraction, la rétention et la mobilité et la section 3 portait sur les services d'aide à l'établissement et à l'intégration. La section 4 s'intéressait aux activités et services offerts en français à l'Île. Pour sa part, la section 5 portait sur l'intégration économique. Finalement, la conclusion cherchait à établir un portrait géographique du lieu de résidence des nouveaux arrivants et les invitait à nous faire part de leur perception quant aux services qui ne sont pas offerts ainsi que sur d'autres besoins qui ne seraient pas comblés. Nous avons tenu à nous assurer que les informations fournies par les répondants soient utilisées en conformité avec les lois sur la Protection des renseignements personnels au Canada, sur l'Accès à l'information et la protection de la vie privée de l'Île-duPrince-Édouard ainsi que du Bureau d'éthique de la recherche de UPEI (UPEI Research Ethics Board). Ainsi, les réponses demeurent confidentielles et font partie d'une base de données sécurisée utilisées uniquement à des fins d'étude. Pour éviter l'identification des répondants, les résultats de cette étude ont été analysés uniquement en format de groupe.

Ce qui nous intéresse dans le cadre du présent article, est le fait que, malgré le faible échantillon de répondants, 15 seulement, il est possible de dégager certaines tendances. Ainsi, au niveau des activités culturelles, l'on note que le taux de participation des nouveaux arrivants d'expression française aux activités de la communauté acadienne et francophone est quelque peu problématique. Rappelons que ces activités ont surtout lieu dans les centres scolaires et communautaires et que ces derniers opèrent comme modélisateurs de la culture acadienne sur l'Île. Dans notre sondage, 13 répondants sur les 15 ont affirmé participer aux activités de la communauté en moyenne au moins une fois ou deux par année. Le taux de participation diminue toutefois en fonction de l'intensité de leur participation. Ainsi, trois répondants participeraient aux activités organisées en français par les centres scolaires et communautaires au moins une fois par mois et un seul y participerait de façon hebdomadaire. Pourtant, un participant a affirmé qu'il faudrait "organiser plus d'activités d'intégration. Avoir des activités pour que les nouveaux arrivants comprennent et intègrent la culture acadienne ». Un autre participant ajoute : « il serait bien d'avoir des activités non seulement avec la communauté francophone, mais aussi anglophone pour favoriser l'intégration, la pratique de la langue anglaise ainsi que le partage culturel ». Le lieu qui leur propose des activités en français ainsi que la nature même des activités proposées deviennent contestables, car le tout 
Alternative Francophone vol. 2, 2(2018): 29-47

http://ejournals.library.ualberta.ca/index.php/af

semblerait empêcher l'intégration à la culture acadienne tout comme à la communauté anglophone.

Deuxièmement, ce sondage nous a aussi appris que seulement 3 répondants avaient des enfants d'âge scolaire, et que seulement deux d'entre eux (2) ont choisi une école de langue française pour leurs enfants, 2 ont choisi une école de langue anglaise et 1 une école privée de confession chrétienne. Un de ces répondants a affirmé vouloir que ses enfants fréquentent plutôt une école de langue anglaise, un autre aurait souhaité la même chose pour une école de langue française et un autre aurait souhaité un autre type d'école. Dans le cas des parents qui auraient voulu que leurs enfants fréquentent une école de l'autre langue, ils ne l'ont pas fait. Autrement dit, le répondant qui aurait souhaité envoyer ses enfants dans une école de langue anglaise, les a envoyés dans une école de langue française, et celui qui aurait voulu envoyer ses enfants à l'école de langue française les a envoyés à l'école de langue anglaise. Dans les deux cas, il s'agit de la même raison : les enfants n'avaient pas d'amis dans l'école souhaitée par les parents. La socialisation des enfants a ici eu préséance sur la question linguistique. Le sondage ne dit toutefois pas s'il s'agit de répondants qui fréquentent les activités des centres scolaires et communautaires de façon régulière ou non, ce qui, nous aurions pensé, aurait pu faciliter la socialisation des enfants en français.

Troisièmement, on a également pu en apprendre davantage sur la situation d'emploi des répondants pour être en mesure d'évaluer leur capital d'employabilité sur l'Île. 7 des 15 répondants avaient alors un emploi à temps plein de 30 heures par semaine ou plus, 3 étaient étudiants à temps plein au niveau collégial ou universitaire, 2 étaient travailleurs temporaires et 3 étaient sans emploi. Quoiqu'il s'agisse d'un faible échantillonnage, on remarque que le taux de chômage des nouveaux arrivants d'expression française suggéré par ces réponses, en se situant à $20 \%$, dépasserait de loin celui de l'ensemble de la province qui se situe autour de $8 \%$. 6 répondants ont affirmé avoir trouvé assez difficile (4) ou très difficile (2) de trouver un emploi qui permet l'utilisation de leurs compétences à l'Île. La difficulté majeure viendrait, dans le cas de 5 répondants, du fait qu'il n'y ait pas beaucoup d'emplois qui correspondent à leurs qualifications et, dans le cas de 5 répondants, de leur utilisation de la langue anglaise. Près de la moitié des répondants affirment toutefois être très satisfaits (3) ou satisfaits (4) de leur emploi actuel. Sept répondants croient que leur

\footnotetext{
8 Pour les données relatives au chômage en 2017, voir Statistique Canada, tableau 282-0087.
} 
Alternative Francophone vol. 2, 2(2018) : 29-47

http://ejournals.library.ualberta.ca/index.php/af

emploi actuel leur permet d'utiliser beaucoup (5) ou un peu (2) les compétences qu'ils ont acquises dans leur pays.

Ces réponses révèlent un net besoin de développer les compétences linguistiques en anglais. Le caractère «bilingue » recherché chez les nouveaux arrivants d'expression française potentiels lors du recrutement à l'étranger se pose désormais comme une priorité. À ce niveau, la nouvelle stratégie du RIF montre une adaptation à la réalité des nouveaux arrivants suite à ce sondage. Ainsi, lors de ses activités de promotion liées à Destination Canada ou encore à Destination Acadie, la CIF met maintenant l'accent sur les secteurs économiques à l'intérieur desquels le manque de main d'œuvre se fait sentir. Un partenariat stratégique avec le RDÉE et un autre avec le Collège de lîle permet de mettre en valeur les emplois disponibles dans les domaines de l'agriculture et de l'éducation à la petite enfance. Il demeure cependant un fait : la connaissance de l'anglais est essentielle pour vivre à l'île.

\section{Conclusion}

Qu'est que tout cela représente pour les nouveaux arrivants d'expression française? S'ils ont choisi l'Île-du-Prince-Édouard, leur sera-t-il possible de choisir le français pour s'épanouir et faire grandir leur famille? Provenant de pays aussi variés que la Belgique, la France, Haiti, l'Iran, ou encore le Maroc, le nouvel arrivant se retrouve dans une petite communauté qui se dit acadienne et francophone, et qui mise donc à la fois sur son ethnie, acadienne, et sa langue, française. Voilà les bases d'un discours sur soi que l'on veut ouvert sur l'autre et qui propose à la fois une approche ethnique et une approche civique (Traisnel, Violette et Gallant 2013, 18).

Ces deux approches peuvent-elles se concilier sur l'Île? Le discours du mouvement communautaire qui s'est rassemblé autour de la CIF dans le dossier de l'immigration pour constituer le RIF se veut inclusif dans son approche civique, mais est porteur de sérieuses apories. La structure que s'est donnée le RIF, sa vision et son mandat oscillent constamment entre la notion d'un « Nous » ethnique et celle d'un « Nous » qui se voudrait civique, au risque de ne pas dépasser les limites de la mémoire. Cependant, il ne s'agit ni de « rejeter la mémoire ni [de] s'y soumettre, [mais plutôt de] l'assumer, [...] en faire une expérience d'historicité » (Thériault 118). Si les seuls lieux pour utiliser la langue française sur l'île demeurent en marge de la société insulaire et exigent des efforts d'adhésion à un pacte politique lié à un groupe pour sauvegarder sa langue, il sera difficile pour la CAFÎPÉ de renverser les tendances de la 
Alternative Francophone vol. 2, 2(2018) : 29-47

http://ejournals.library.ualberta.ca/index.php/af

décroissance démographique et connaître du succès au niveau de l'immigration d'expression française.

Comme l'a montré Isabelle Violette, la transformation de l'Acadie comme société d'accueil renferme son lot de contradictions et de conflits (148), mais, à la différence de l'Acadie du Nouveau-Brunswick, l'Acadie insulaire commence seulement à apprivoiser les outils idéologiques et politiques pour faire face à cette transformation d'une façon efficace. Il lui reste entre autres à proposer des lieux de partage publics de la parole qui dépassent les dimensions festives et mémorielles pour présenter au nouvel arrivant un véritable pacte qui ne se nourrit pas exclusivement de la mémoire. Des efforts en ce sens existent déjà, mais ils sont loin de constituer une stratégie collective de réseautage. Il ne s'agit pas de brûler la souche acadienne, comme dirait Gérard Bouchard en parlant de la souche québécoise, mais d'y greffer une variante moderne qui s'éloigne de la nostalgie (Bouchard 2012).

La faible utilisation du français dans les espaces publics pose aussi la question de la légitimité de la langue française sur l'île. Les Acadiens expliquent leurs origines, leurs racines, commémorent et célèbrent les moments forts de leur histoire, leur réussite et leur résilience en français le plus souvent seulement entre eux. L'on approche les potentiels immigrants de langue française intéressés à venir s'établir dans l'île en prenant soin de leur faire voir l'importance de la langue anglaise. Pour ne plus être dominé socialement, économiquement et politiquement, l'Acadien de l'île concède sa langue maternelle. À moins que le français ne devienne une langue publique sur l'île et quitte les quelques îlots de résistance dans lesquels il évolue, les nouveaux arrivants sont appelés à s'angliciser à moyen terme, car, devant l'Autre, le quotidien se vit dans la langue de la majorité. Mais il s'agit peut-être d'une façon de réduire les effets de la différence inhérente à l'utilisation d'une langue en situation minoritaire. La dichotomie dominants-dominés se verrait ainsi à tout le moins atténuée. Le faire-société en situation minoritaire constituerait à participer au progrès de la majorité en en faisant partie. La diversité se perdrait alors dans l'unicité du groupe majoritaire, la sphère économique faisant disparaittre peu à peu la sphère culturelle, ce qui tend à faire du français un outil dans la promotion du bilinguisme.

La mutation identitaire des organismes communautaires de langue française de l'Île-du-Prince-Édouard repose sur une meilleure compréhension des attentes de la communauté en matière de croissance démographique, mais aussi des motivations du nouvel arrivant d'utiliser la langue française. La 
polysémie sémantique qui est offerte dans la description même de la communauté, acadienne et francophone, et dans sa stratégie de croissance démographique montre en fait que « Nous » devrait être un projet insulaire, un projet qui réunit Acadiens, francophones et francophiles. Mais ce projet oscille encore entre une description passéiste donnant préséance aux Acadiens issus de la Déportation, francophones et catholiques (SSTA et sa mission) et un discours moderne dont le RIF serait l'emblème. Le changement de nom de la seule institution d'enseignement postsecondaire de langue française est dans ce sens un fort indicateur des changements à venir. L'ancien nom - Collège Acadie Îledu-Prince-Édouard - était perçu comme «quelque-chose d'unique à la communauté acadienne alors que l'établissement cherche au contraire à élargir ses horizons» (ICI 2016). Pour communiquer une ouverture et devenir rassembleur, le Collège Acadie-ÎPÉ est devenu le Collège de l'île à l'automne 2016, un peu à l'image du Carrefour d'Intégration Rurale Évangéline qui est devenu la Coopérative d'intégration francophone en 2010.

Dans une province où la langue française est en situation minoritaire, chaque mot, chaque adjectif revêt une importance capitale. La réalité que l'on croit désigner avec justesse peut souvent faire référence à d'autres concepts pour le nouvel arrivant et devenir une source d'exclusion. Mais surtout, il faut arriver à sortir le français des espaces clos de la maison et des organismes communautaires et maximiser son utilisation publique. Il y aurait alors lieu de penser à un entre-deux, comme le propose Régine Robin à propos du Québec : un «interstice, le jeu d'une identité ne craignant ni l'entame ni l'indétermination» $(2011,52)$. En sortant des cadres des centres scolaires et communautaires tout en y prenant racine, une Acadie véritablement insulaire pourrait jeter les bases d'une identité qui dépasse la mémoire acadienne et les dimensions utilitaires de la langue et proposer un «Nous» auquel Acadiens, francophones et francophiles pourraient adhérer, parce que véritablement ouvert, public et non marginal. Le lien de l'unicité de ce « Nous » serait dès lors territorial, insulaire, véritablement ouvert à l'Autre, et non seulement mémoriel. 
Alternative Francophone vol. 2, 2(2018): 29-47

http://ejournals.library.ualberta.ca/index.php/af

\section{Bibliographie}

Arsenault, Georges. Les Acadiens de l'Île: 1720-1980. Moncton : Éditions d'Acadie, 1987.

---. Contes, légendes et chanson de l'Île-du-Prince-Édouard. Moncton: Éditions d'Acadie, 1998.

Beausoleil, Claude et Gérald Leblanc. La Poésie acadienne. Anthologie. Moncton/Trois-Rivières: Éditions Perce-Neige/Écrits des Froges, 1999.

Belkhodja, Chedly. D'ici et d'ailleurs. Regards croisés sur l'immigration. Moncton: Éditions Perce-Neige, 2011.

Belkhodja, Chedly et Michelle Vatz Laaroussi (sous la direction de). Immigration en dehors des grands centres : enjeux, politiques et pratiques dans cinq états fédéraux. Paris : Éditions L'Harmattan, 2012.

Bouchard, Gérard. L’interculturalisme : un point de vue québécois. Boréal : Montréal, 2012.

Boulogne, Charles-Damien. Saint Thomas-d'Aquin ou le génie intelligent. Paris : Nouvelles éditions latines, 1965.

Bourdieu, Pierre. Raisons pratiques : sur la théorie de l'action. Paris : Seuil, 1994.

Canadian Parents for French. Enrollment trends, French Immersion, 2016-2017, 2016.

http://pei.cpf.ca/enrollment-trends-pei-french-immersion-and-corefrench/. Consulté le 12 mai 2017.

Coopérative d'intégration francophone. Rapport annuel 2014-2015. Summerside : 2015. http://tonile.ca/images/rapport_annuel_20142015-2.pdf. Consulté en le 12 mai 2017.

Cormier, Angie. A recruitment and attraction strategy for bringing Francophone immigrants to PEI. EMBA Signature Project. UPEI, 13 novembre, 2013. 
Division de l'analyse du marché du travail, Service Canada, Île-du-PrinceÉdouard. «Bulletin sur le marché du travail - ̂̂le-du-Prince-Édouard : Janvier $2015 »$, http://www.edsc.gc.ca/fra/emplois/imt/ publications/bulletins/ipe/jan2015.shtml, février 2015. Consulté le 12 mai 2017.

François, Frédéric. «Le social incorporé dans l'individu et sa représentation dans le discours. Bourdieu, l'habitus et l'(auto)biographie». Langage et société, $\mathrm{n}^{\text {os }}$ 121-122, 2007, pp. 45-56.

Gallant, Melvin. À la conquête de l'île Saint-Jean. Tracadie-Sheila : Éditions La Grande Marée, 2016.

---. Le Métis de Beaubassin. Lévis : Éditions de la Francophonie, 2009.

Gallant, Nicole. «De l'ouverture à l'inclusion : immigration et identité en milieu francophone minoritaire ", dans Chedly Belkhodaj (dir.), Canadian Issues/Thèmes canadiens. Immigration et diversité au sein des communautés francophones en situation minoritaire, printemps 2008 : 43-46.

Groupe de travail conjoint Communauté acadienne et francophone Gouvernement de l'Île-du-Prince-Édouard (2013). Rapport, avril.

Ici Radio-Canada. «Le collège de langue française de l'Î.-P.-É. change de nom ». Publié le lundi 12 décembre 2016.

http://ici.radio-canada.ca/nouvelle/1005296/le-college-de-languefrancaise-de-li-p-e-change-de-nom. Consulté en ligne le 12 mai 2017.

Johnson, Marc. Transmission de la langue française aux jeunes francophones en situation minoritaire. Théorie et stratégie. Rapport final. Une initiative de la Direction générale des langues officielles, ministère du Patrimoine canadien. Gatineau, 2015.

Lavoie, Carlo, Audrey-Anne Jean, Jean-Phylippe Provencher et Marianne Tremblay-Abel. Recrutement et intégration des nouveaux arrivants de langue française à l'île-du-Prince-Édonard. Rapport de recherche. London (Ontario) : Voies vers la Prospérité, 2016. http://p2pcanada.ca/wpcontent/blogs.dir/1/files/2016/05/Recrutement-et-integration-aIPE.pdf. Article consulté en ligne le 23 avril 2017. 
Alternative Francophone vol. 2, 2(2018) :29-47

http://ejournals.library.ualberta.ca/index.php/af

Lavoie, Carlo. Atouts et lacunes de la communauté acadienne et francophone et les besoins des nouveaux arrivants d'expression française à l'Île-du-Prince-Édouard: un rapport exploratoire. Rapport de recherche. Charlottetown: Coopérative d'intégration francophone de l'Île-du-Prince-Édouard, 2015.

Lockerby, Earl. La Déportation des Acadiens de l'Âle-du-Prince-Édonard. Montréal : Éditions au Carré, 2010.

Paré, François. Littératures de l'exiguïté. Ottawa : Le Nordir, 2001.

Paul (Gilbert Buote). Placide, l'homme mystérieux à New York. Moncton : Bouton d'or Acadie, 1999.

Robin, Régine. Nous autres, les autres. Montréal : Boréal, 2011.

---. La Québécoite. Montréal : Québec-Amérique, 1983.

SSTA. Comité sur la planification stratégique 2016-2026. Planification stratégique 2016-2026. Communauté acadienne et francophone de l'Île-du-PrinceÉdouard. Plan de travail. Février 2016.

https://ssta.org/images/PDF/CPS $\% 20$ Plan $\% 20 \mathrm{de} \% 20$ travail $\% 20 \mathrm{fv}$. \%202016.pdf. Consulté le 12 mai 2017.

Statistique Canada. Série "Perspective géographique », Recensement de 2011. Produit no 98-310-XWF2011004 au catalogue de Statistique Canada. Ottawa, Ontario. Produits analytiques, Recensement de 2011. Version mise à jour le 24 octobre 2012.

Statistique Canada, CANSIM, tableau 282-0087 et produit no 71-001-XIF au catalogue. Dernières modifications apportées : 2017-05-05.

Thériault, Joseph Yvon. Faire société. Société civile et espaces francophones. Sudbury: Prise de parole/Agora, 2007.

Traisnel, Christophe, Isabelle Violette et Nicole Gallant. «Les minorités francophones canadiennes face à l'immigration: particularismes et représentations de la diversité ethnoculturelle dans les milieux associatifs ", dans Minorités linguistiques et société/Linguistic Minorities and Society, $\mathrm{n}^{\circ}$ 2, 2013, pp. 9-28.

(c) (i) (9) 
Alternative Francophone vol. 2, 2(2018) : 29-47

http://ejournals.library.ualberta.ca/index.php/af

Violette, Isabelle. «L'immigration francophone en Acadie : langue, nation et minorité », dans Minorités linguistiques et société/Linguistic Minorities and Society, $\mathrm{n}^{\circ} 4,2014$, pp. 126-153. 